\title{
A Methodology to Create 3D Body Models in Motion
}

\author{
Eduardo Parrilla, Ana-Virginia Ruescas, Juan-Antonio Solves, \\ Alfredo Ballester, Beatriz Nacher, Sandra Alemany ${ }^{(\bowtie)}$, \\ and David Garrido
}

Instituto de Biomecánica (IBV), Universitat Politécnica de València, Camino de Vera s/n, Edificio 9C, 46022 Valencia, Spain

\{eduardo. parrilla, ana. ruescas, juanantonio. solves,

alfredo.ballester, beatriz. nacher,

sandra.alemany\}@ibv.org

\begin{abstract}
Size, shape and posture are fundamental features of digital human models (DHM) to obtain accurate virtual simulations of the ergonomics of products and environments. Research on 3D body scanning, processing and modelling have enabled the generation of avatars representing specific populations and morphotypes in standing and seated postures being the basis to define size and shape of DHM. Posture is implemented with biomechanical models of the human movement. Most of the research is focused on posture control and movement tracking to analyze the variability in different contexts (e.g. driving, performing a working task). Motion capture technology used for this purpose, requires a limited number of sensors or reflective markers attached to the body according to the definition of body segments. 3D body scanning and motion capture are both technologies currently used to analyze human body shape and biomechanics to apply it to enhance digital human models. These technologies may converge on the so-called temporal 3D scanners or 4D scanners, a new technology recently developed to scan the body in motion. With this technology, it is possible to obtain sequences of dense 3D point clouds representing the movement of the body. In this paper, a novel methodology to create realistic 3D body models in motion is proposed. This method is supported by a new 4D scanning system (Move $4 D$ ) and a data driven-model. Move4D is a modular photogrammetry-based 4D scanning system. It consists of a set of 12 synchronized modules to scan full bodies with texture in motion. It can capture up to $180 \mathrm{fps}$ with a resolution of $2 \mathrm{~mm}$. The algorithms have been conceived and optimized to automatically process the series of raw point clouds captured. They rely on a data-driven body model including shape, pose and soft-tissue deformation trained with a large database and a deep learning model. The process is fully automatic and does not require any interactive landmarking or revision. The 3D outcome of this methodology is one noise-and artefact-free watertight mesh per frame and a model of shape, pose and soft-tissue that can be rigged with a 23-joint skeleton. This type of outcome permits their use for many applications such as simulations, augmented and virtual reality (AR/VR) or biomechanical analysis purposes.
\end{abstract}


Keywords: Anthropometry $\cdot$ Temporal scanning 4D $\cdot$ Data-driven $\cdot$ Body model $\cdot$ Human motion $\cdot$ Marker-less $\cdot$ Motion analysis $\cdot$ Image processing $\cdot 3 \mathrm{D}$ body model $\cdot$ Shape $\cdot$ Pose $\cdot$ Soft-tissue deformation

\section{Introduction}

Human digital models (DHM) are digital patterns that represent different characteristics of humans, being the size, shape and posture some of the most important in the field of ergonomics.

On the one hand, anthropometric models are commonly used to define the dimensions and the shape of the products and workplaces [1]. The use of threedimensional (3D) body scanners has improved the capture of anthropometric data in terms of both, accuracy and time spent. In addition, the research on processing and 3D body modelling has allowed the generation of anthropometric data collections. This data is very useful in simulation applications and to obtain avatars that represent specific populations and morphotypes. However, there are some applications that need to take the anthropometric measurements in different postures (e.g. ergonomic wearing comfort). In these cases, a limited number of $3 \mathrm{D}$ scans that describe the movement to study are performed step by step, but it is not clear whether the results can illustrate the real dynamic changes of the body [2].

On the other hand, biomechanical models focus on the mechanical and dynamic properties of the human body. Most of the research is focused on posture control and movement tracking to analyze the variability in different contexts (e.g. driving, performing a working task). Posture and movement detections are important challenges for the generation of these models [1]. Biomechanical models are often satisfied with three important percentiles for each gender, but nowadays, it is preferred to capture movements from real subjects. The most used technology to record movement requires sensors or reflective markers attached to the body according to the definition of body segments. It is also remarkable that the most precise marker-based capture method requires a human model previously adapted to the anthropometric characteristics of the real subject. However, the use of markers is invasive and may interfere with the measured movements. So that, there have been developed optical detection marker-less systems in this area [3].

As anthropometric models can be improved by considering posture dependencies and biomechanical models can be improved adapting them to anthropometric characteristics, merging movement and anthropometric capture technologies can lead with the previous dependencies. The so-called temporal 3D scanners or 4D scanners implements movement and anthropometric captures combination. These systems allow to obtain sequences of dense 3D point clouds that represent the movement of the body. In this article, a new methodology is proposed to create realistic 3D body models in motion. This method is compatible with a new 4D scanning system (Move 4D) and a datadriven model. 


\section{Methodology to Create 3D Bodies in Motion: Move 4D}

Move 4D (Fig. 1) is a modular photogrammetry-based 4D body scanner developed by the Institute of Biomechanics of Valencia (IBV) [4].

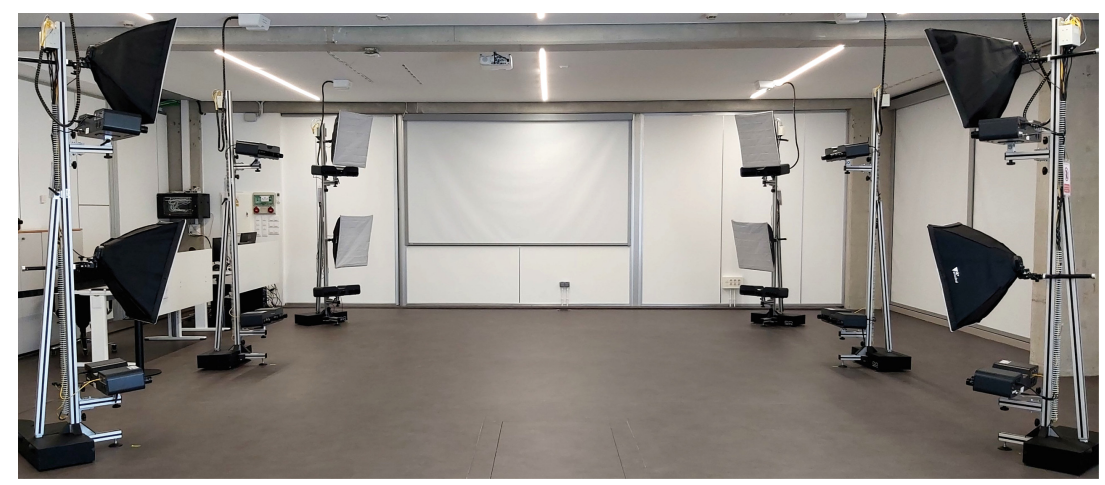

Fig. 1. Move $4 D$ at Human Analysis Lab (HAL) in the Institute of Biomechanics of Valencia.

\subsection{Move 4D Features}

Move $4 D$ consists of a set of synchronized modules to scan body parts or full bodies with texture in motion, a control unit and a processing software. Each module (Fig. 2) consists of a pair of infrareds (IR) cameras, an IR projector, a color (RGB) camera and a processing unit.
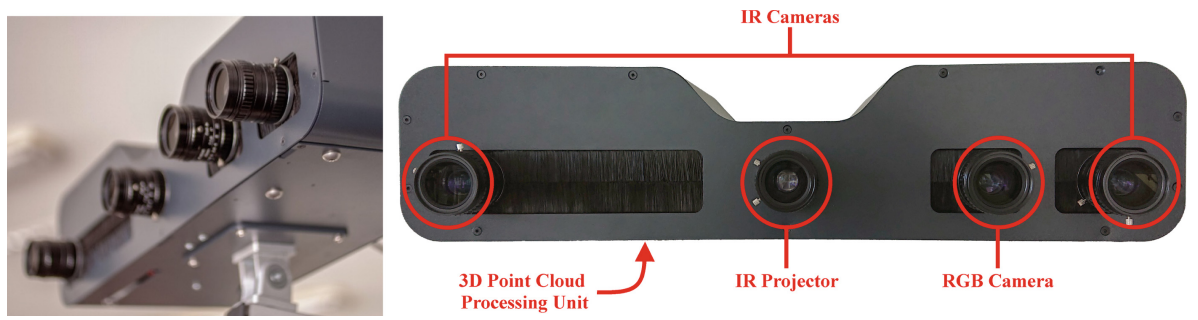

Fig. 2. Detail of Move $4 D$ module unit.

The equipment is portable and its modularity permits to customize the scanning space. It can be installed an unlimited number of modules and, also, other devices (Fig. 3) can be connected to it to obtain additional synchronized biometric signals (e.g. thermal cameras, RGB cameras network or force platforms).

A typical full body configuration of 12 synchronized modules permits a scanning space of $2 \mathrm{~m}$ width, $2 \mathrm{~m}$ depth and $3 \mathrm{~m}$ height providing a minimum spatial resolution of $1 \mathrm{~mm}$. Depending on its configuration (Table 1) it can capture up to $180 \mathrm{fps}$. 


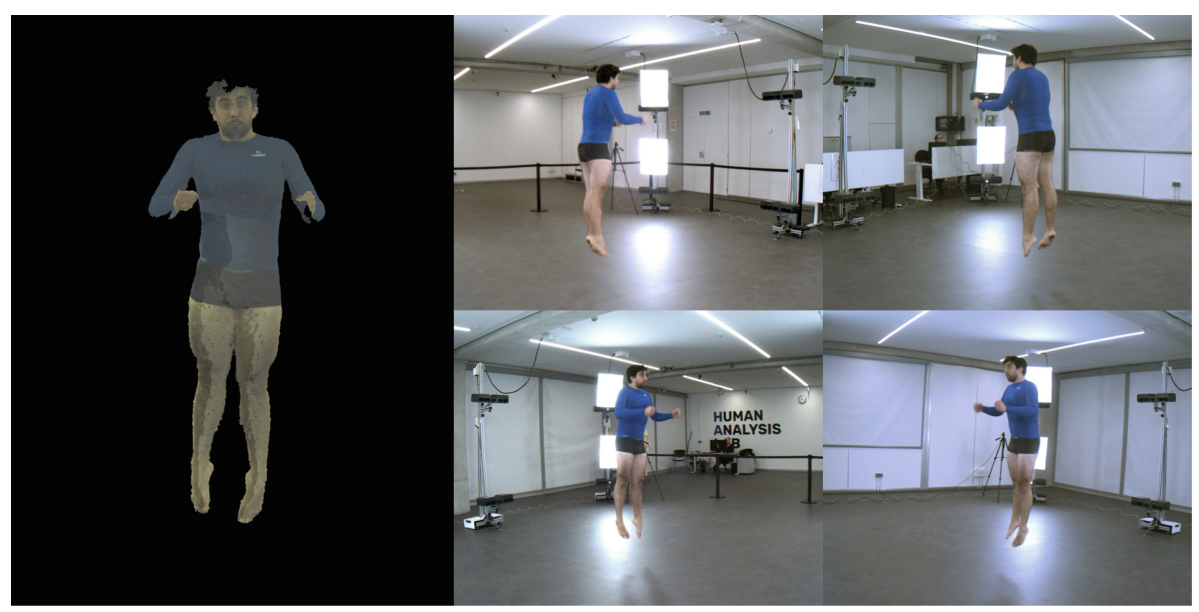

Fig. 3. RGB cameras network synchronized with Move 4D. Left: dense 3D point cloud from Move $4 D$. Right: corresponding images from 4 RGB cameras network.

Table 1. Move $4 D$ technical specifications.

\begin{tabular}{l|l|l}
\hline Characteristics & Values & Notes \\
\hline Optic unit resolution & $1-2 \mathrm{~mm}$ & Corresponding to high - medium resolution \\
\hline Maximum captures frequency & $90-180 \mathrm{fps}$ & Corresponding to high - medium resolution \\
\hline Scanning time & $1 \mathrm{~ms}$ & \\
\hline Footprint & $5.5 \times 5.5 \mathrm{~m}$ & Corresponding to 12 units configuration \\
\hline External synchronism & $\mathrm{I} / \mathrm{O}$ & Trigger and synchro input/output (I/O) \\
\hline Capture & & 3D data and corresponding color (texture) \\
\hline Lighting & & Inbuilt lighting system \\
\hline
\end{tabular}

The software has been conceived and optimized to automatically process the sequence of full bodies 3D point clouds captured, avoiding any interactive landmarking or revision. The implemented process relies on deep learning and a data-driven body model that considers shape, pose and soft-tissue deformation.

\subsection{Move 4D Outcomes}

The 3D outcome of this methodology is one noise- and artefact-free watertight dense mesh (99 K tri faces) per frame (Fig. 4). The 3D meshes of one sequence can be provided with point-to-point correspondence ( $50 \mathrm{~K}$ landmarks) and can also be rigged with a 23-joint skeleton. 


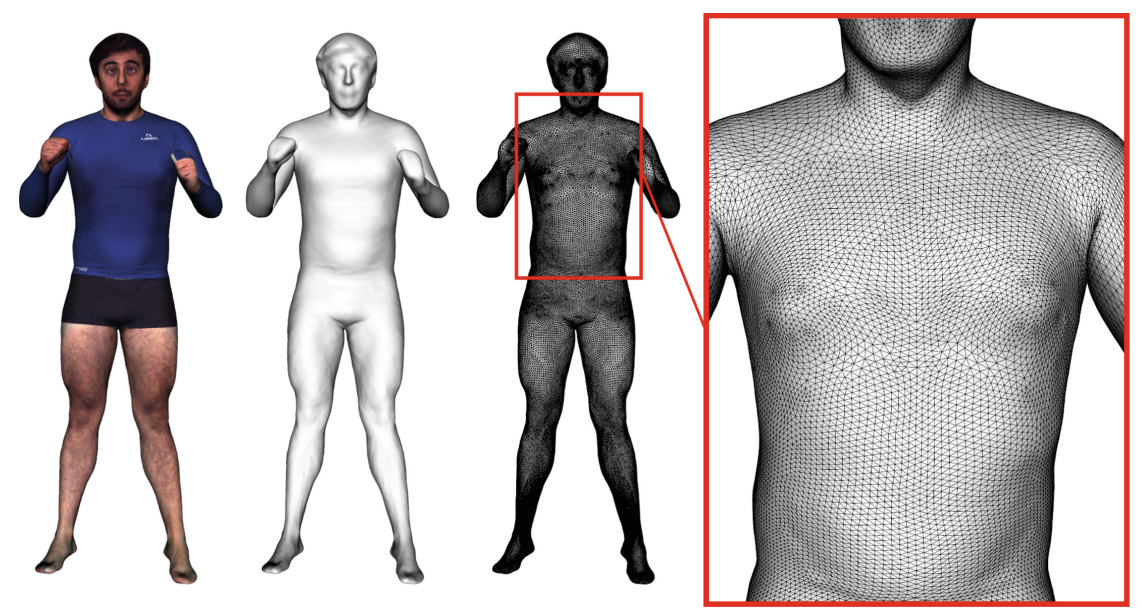

Fig. 4. Noise- and artefact-free watertight dense mesh with texture and detail of landmarks.

This type of outcome can be suitable for applications such as CAD, simulation, $\mathrm{AR} / \mathrm{VR}$, animation, etc. The format of the 3D outcomes enables the tracking of points along the motion sequence captured for biomechanical analyses purposes in sports health applications and health-related assessments.

\section{Future Research Based on 3D Body Avatars in Motion}

4D scanning technology provides a new way to capture information of the body surface in motion. Additional insights and features could be added to the current knowledge of body anthropometry and biomechanics to enhance current DHM. Addition research is required to analyze the reliability of this methodology compared to the current validated methods.

A roadmap of integration of both body shape and biomechanical models it necessary to optimize new research based on the proposed methodology. In this line, and considering the current stage of development of the new technology and methods to create 3D body avatars in motion, it is important to analyze the potential of standardization. A standard 3D body model defined with a smart mesh topology to automatically separate body parts, integrated with a standard biomechanical model represented by the internal skeleton (e.g. ISB models, or ISO), is essential to take advantage of the current state of the art and progress in the creation of 3D body models in motion.

Acknowledgment. The research presented in this paper have been developed within the projects IMDEEA/2020/85 and MDEEA/2020/87. Funding requested to Instituto Valenciano de Competitividad Empresarial (IVACE), call for proposals 2020 for Technology Centers of the Comunitat Valenciana, co-funded by ERDF Funds, EU Operational Program of the Comunitat Valenciana 2014-2020. 


\section{References}

1. Scataglini, S., Paul, G.: DHM and Posturography. Academic Press, London (2019)

2. Zakaria, N., Gupta, D.: Anthropometry, Apparel Sizing and Design. Woodhead Publishing, Cambridge (2019)

3. Liberadzki, P., Adamczyk, M., Witkowski, M., Sitnik, R.: Structured-light-based system for shape measurement of the human body in motion. Sensors 18, 2827 (2018). https://doi.org/10. 3390/s18092827

4. Parrilla, E., Ballester, A., Parra, P., Ruescas, A., Uriel, J., Garrido, D., Alemany, S.: MOVE 4D: accurate high-speed 3D body models in motion. In: Proceedings of 3DBODY.TECH 2019, Lugano, Switzerland, 22-23 October 2019, pp. 30-32 (2019). https://doi.org/10.15221/ 19.030 